\title{
The role of genes in defining a molecular biology of PTSD
}

\author{
Rachel Yehuda ${ }^{\mathrm{a}, *}$, Karestan C. Koenen ${ }^{\mathrm{b}}$, Sandro Galea ${ }^{\mathrm{c}}$ and Janine D. Flory ${ }^{\mathrm{a}}$ \\ ${ }^{a}$ Department of Psychiatry, James J. Peters Veterans Affairs Medical Center, Bronx, New York, USA and \\ Department of Psychiatry, Mount Sinai School of Medicine, New York, NY, USA \\ ${ }^{\mathrm{b}}$ Departments of Society, Human Development and Health and Epidemiology, Harvard School of Public Health, \\ Boston, MA, USA and the Harvard Center on the Developing Child, Cambridge, MA, USA \\ ${ }^{\mathrm{c}}$ Department of Epidemiology, Columbia Mailman School of Public Health, New York, NY, USA
}

\begin{abstract}
Because environmental exposure to trauma is the sine qua non for the development of Post Traumatic Stress Disorder (PTSD), the recent focus on genetic studies has been noteworthy. The main catalyst for such studies is the observation from epidemiological studies that not all trauma survivors develop this disorder. Furthermore, neuroendocrine findings suggest preexisting hormonal alterations that confer risk for PTSD. This paper presents the rationale for examining genetic factors in PTSD and trauma exposure, but suggests that studies of genotype may only present a limited picture of the molecular biology of this disorder. We describe the type of information that can be obtained from candidate gene and genomic studies that incorporate environmental factors in the design (i.e., gene - environment interaction and gene-environment correlation studies) and studies that capitalize on the idea that environment modifies gene expression, via epigenetic or other molecular mechanisms. The examination of epigenetic mechanisms in tandem with gene expression will help refine models that explain how PTSD risk, pathophysiology, and recovery is mediated by the environment. Since inherited genetic variation may also influence the extent of epigenetic or gene expression changes resulting from the environment, such studies should optimally be followed up by studies of genotype.
\end{abstract}

\section{If PTSD is a condition precipitated by environmental exposure, why examine genes?}

For at least two decades after the diagnosis of PTSD was established in 1980, it would have been unheard of to propose that genes might be involved in the etiology or pathogenesis of this condition. PTSD was initially defined as a disorder that resulted from exposure to a traumatic environmental event. The diagnosis was designed to describe universal effects of extreme stress that linger even after the stressor is removed. The theoretical contribution that this made to the biopsychosocial model of mental illness was the realization that the effects of an environmental event are not limited to initial exposure to the event, but can also persist for years and even decades. These long-term effects were conceptualized as being a function of the intensi-

\footnotetext{
*Corresponding author. E-mail: Rachel.Yehuda@va.gov.
}

ty and severity of the event that precipitated the initial symptoms.

The implication of the PTSD diagnosis was that the adverse effects of exposure to chronic stressors (e.g., illness, family conflict, or financial pressures) could be alleviated or even eliminated if the challenge to the person was removed (and hence were not precipitants of PTSD). In contrast, the effects of exposure to lifethreatening events like interpersonal violence, combat, and accidents that caused intense fear, helplessness and horror were not only persistent, but were of a specific and universal nature. The basic phenomenology of PTSD asserted that following trauma exposure, the survivor experiences unwanted, uncontrollable memories of the event that generate physical and emotional responses resembling the fear responses that occur at the time of exposure to the traumatic event. These memories result in avoidance behavior and hyperarousal, and ultimately lead to social, occupational or interpersonal dysfunction. Because exposure to fearful situations 
was thought to precipitate PTSD, the agenda for the first generation of neuroscience-based studies in PTSD was to examine the neural, chemical, and hormonal correlates of fear so as to better target the relevant biological systems involved in its pathophysiology. The idea of identifying tangible evidence of a lingering disturbance in stress-related neurobiology would serve not only to identify treatment targets, but also to validate the existence of the PTSD diagnosis.

There were two major observations in the 1990's that changed the trajectory of biological studies in PTSD. The first observation came from a series of reports showing that measures of cortisol and other aspects of hypothalamic-pituitary-adrenal (HPA) axis regulation in PTSD did not correspond to what would have been predicted on the basis of stress theory. The most striking finding was that of lower cortisol in people with PTSD, compared to those without PTSD [1]; (see [2] for a review), which was unexpected because this hormone is classically thought to be elevated in the context of extreme stress and fear. At first these findings were considered paradoxical because they not only contradicted the prediction that cortisol levels would be elevated in a stress disorder, but also because they were different than they would be in conditions that are frequently comorbid with PTSD, such as in major depressive disorder. However, the second series of observations substantially clarified the results from the hormonal studies, albeit indirectly. From epidemiologic studies it became clear that only some persons exposed to trauma develop and maintain PTSD while others do not [3-5].

The absence of PTSD in some trauma-exposed persons challenged initial conceptions of PTSD as a normative response to extreme stress. This helped free the field from the tyranny of needing to hypothesize that PTSD biology would be analogous to biological alterations seen in response to stress and fear. The observation from epidemiological studies that trauma exposure alone does not fully determine either the development of, or recovery from, PTSD provided the justification for evaluating pre-traumatic risk factors, and for considering whether characteristics that differentiate trauma survivors with and without PTSD reflect risk for PTSD [6]. Whereas previously it was important to understand the biological changes that result from exposure to environmental events, it was now clear that such responses might have earlier antecedents that affect such responses.

Together, then, biological and epidemiological studies also clarified that PTSD does not represent a univer- sal response to a major environmental event, but rather a specific phenotype, expressed in the presence of an environmental stressor, that is characterized by a series of biological events that do not permit, or in fact directly impair, reinstatement of physiologic homeostasis or recovery from trauma [2,7]. If PTSD represents a failure to recover from the normal effects of trauma, then the pathophysiology of PTSD might be explained by individual differences in modulators of the response to stress [8]. Delineating the contributions of such differences to the development of PTSD requires a broader assessment of vulnerability than is currently practiced and will ideally include genotypic and molecular factors, in addition to clinical and functional ones.

In this paper, we provide a justification for examining molecular genetics and clarify current experimental approaches that can be used to identify genetic and other molecular alterations in PTSD with the goal of identifying mechanisms that explain PTSD risk, pathophysiology, and recovery. Our purpose is to specify the information that will be yielded by examining gene $\mathrm{x}$ environment $(\mathrm{GxE})$ interactions in PTSD versus approaches that will inform the enduring effects of environment on genes. We describe the three major categories of molecular genetic studies of PTSD so as to identify some of the complexities of considering genes in the study of PTSD. Our intention is to emphasize differences between studies designed to examine genotype by environment interactions in PTSD versus those that aim to elucidate how environmental experiences such as trauma can produce PTSD, and examine the implications for a molecular biology of PTSD.

\section{Genetic vulnerability associated with PTSD}

The idea that the presence of a specific gene variant (allele) could contribute to individual differences in response to a traumatic event has been embraced by the mental health community, even though this idea is fairly radical in the context of a disorder that is thought to be brought on by exposure to an extremely traumatic event. This may, in part, be related to compelling evidence from twin studies that genetic factors contribute to the risk for PTSD (e.g [9-11] see [12] for review). This was first demonstrated in a cohort of combat veterans [13], in which the risk for developing PTSD after trauma exposure was significantly greater for monozygotic (non-combat exposed co-twin) than for dizygotic twins of PTSD-affected probands. In a populationbased sample of twins [14], not only was PTSD more 
likely to be concordant in monozygotic twins, but there was also a genetic association with exposure to violence (but not accidents or disaster). It was concluded that the genetic risk for assault trauma could reflect personality traits - anger or irritability - that increased the likelihood for assault.

Below we review several approaches to identifying a genetic basis for PTSD while noting that at the writing of this review few genes have been linked with PTSD. The paucity of genetic studies in PTSD may reflect the complexity involved in executing research that will provide unambiguous results. That underlying genotypic vulnerability for PTSD can only be expressed following trauma exposure limits the practicality of family-based linkage approaches. Sources of heterogeneity owing to type of exposure presents a dilemma concerning whether to focus on PTSD from a wide range of events, which may themselves involve genetic risk factors [10], or to limit investigations to a particular subgroup with common exposure (e.g., veterans). In considering the appropriate "case-controls" for subjects with PTSD, it is not clear whether to match comparison subjects on the basis of exposure type or other factors such as race, which may also constitute risk factors and, if controlled for, may obscure other genetic associations. The necessity of actively recruiting subjects with PTSD without regard for whether they are representative of the normal population presents a problem of determining the variables on which to match comparison subjects. For example, comparison subjects may possess the genetic vulnerability for PTSD but lack exposure to a traumatic event. Without the ability to examine different levels of trauma exposure from within the context of a common random event affecting an entire population and resulting in different outcomes with respect to PTSD, it is not feasible within the confines of a single study to generate interpretable data.

\section{Gene association studies in PTSD}

One way to determine whether genes are involved in PTSD risk or pathophysiology is to identify allelic variants of genes that might be associated with PTSD. This can be done by examining specific genes that are hypothesized to represent the origin of a more downstream, observed biological alteration. Alternatively, gene variants can be identified by examining [nearly] the entire genome simultaneously. Candidate gene association studies represent the approach most commonly used in the field of PTSD genetics thus far. Such studies examine genotype variations at specific loci on a gene or genes and compare genotype or allelic frequencies between people who do versus do not develop PTSD in a group of similarly exposed trauma survivors. The alleles (i.e., alternative forms of genetic variation - or polymorphisms - at a specific locus) usually take one of two forms: single nucleotide polymorphisms (SNPs), in which the genetic variation occurs at the nucleotide (base pair) level; and variable number of tandem repeats (VNTRs), in which the genetic variation occurs in the number of repetitive sequences (i.e., a length polymorphism).

The most compelling reason for using a candidate approach is that this is a hypothesis testing approach that bases the selection of specific genes on known biological and psychological correlates of PTSD and PTSD risk obtained from prior research on aspects of the biological response to stress, or to biological processes that underlie individual differences in stress vulnerability. For this reason, should different allelic variations of the gene be associated with PTSD, the findings have biological plausibility and are less likely to be viewed as spurious.

However, it is often difficult to identify one or just a few genes that might be related to a complex behavior, neural circuit, or functional system, even if that system is known to be altered in a condition. Indeed, as noted above, one of the most well-developed areas of biological inquiry of PTSD risk is the study of cortisol and the HPA axis. The HPA axis is the major constituent of the neuroendocrine response to acute and chronic stress. In response to stress, the parvocellular neurons of the hypothalamus are stimulated to secrete the neuropeptides corticotropin releasing hormone $(\mathrm{CRH})$ and vasopressin (AVP) into the portal vessels system to activate the synthesis and release of adrenocorticotropic hormone (ACTH) from the anterior pituitary. ACTH stimulates the adrenal cortex to synthesize and release gluccocorticoirds (GCs), in particular cortisol. These hormones have a multiplicity of functions, which are necessary for the adaptation to stress [15]. In PTSD the fine-tuned regulation of this HPA axis is disturbed, which is indicated by reduced cortisol levels, but also by an impaired responsiveness of ACTH and cortisol in these patients (see [16] for a review). What makes the study of HPA-axis related genes in PTSD so compelling is that in several prospective, longitudinal studies, lower cortisol levels in the acute aftermath of trauma were associated with either the subsequent development of PTSD, or with the well-established risk factor of prior trauma exposure, and were not necessarily 
associated with posttraumatic PTSD pathophysiology per se [17]. These findings led to the hypothesis that reduced cortisol levels at the time of a trauma may compromise the inhibition of stress-induced biologic responses (e.g., during and following a traumatic event), resulting in a prolonged physiological/emotional distress which would then facilitate the development of PTSD [2]. That cortisol levels are associated with some aspect of pre-exposure vulnerability provides a plausible explanation for discrepant observations in PTSD. That is, it may be that cortisol levels are low only in PTSD associated with specific pre-exposure risk factors, justifying the examination of cortisol functioning in association with PTSD risk [6]. This reasoning has led to several studies examining gene variants in the CRH gene [18], the glucocorticoid receptor (GR) gene [19], and in FKBP5 [20,21], a gene which regulates cortisol-binding affinity and nuclear translocation of GRs. Because the dopaminergic and adrenergic systems are also implicated in stress and fear biology, variation in dopamine regulating genes (e.g., dopamine $\beta$ hydroxylase, dopamine transporter and DRD2 genes) has been investigated in association with PTSD [2228]. Variation in the serotonin transporter gene has been widely studied in association with PTSD [29-31] and in particular in the context of gene by environment studies [32-34] (see below for further discussion of this type of design). The interested reader is referred to recent reviews of genetic association studies in PTSD for further details $[35,36]$.

Investigating the potential upstream effects of cortisol-related alterations is difficult in practice as there are thousands of genes that relate in some manner to the regulation of glucocorticoids and their actions (in endocrine regulation, stress, cognition, etc.). Thus, even though it would be possible to hypothesize in a broad sense that genes that modulate the actions of glucocorticoids will confer risk for PTSD, a candidate gene approach is not practical. At the present time, given the cost associated with surveying the almost entire genome in a case where several hundred or thousand genes might be candidates, it would be expedient to use a genome-wide approach.

The genome wide genotyping approach can be contrasted to the candidate gene approach in that it is a hypothesis generating method that allows identification of a broad set of relevant biological processes that might otherwise not come into consideration using a candidate gene approach. This comprehensive scan of the genome provides the opportunity to identify novel susceptibility factors for the development and persistence of this disorder. It allows the investigator to not restrict the prediction of a gene of interest to the biology that is known today, but rather aids in the identification of yet unknown mechanisms.

Because of the large number of loci surveyed in a genome-wide analysis, large sample sizes are required for such studies. Even with large samples, spurious findings are likely to occur based on individual sampling characteristics. For this reason, it is appropriate to follow up initial observations from a genome-wide analysis with a replication sample to validate the results obtained. A genome-wide analysis is also best combined with an evaluation of functional measures with known or putative associations to PTSD and PTSD vulnerability in order to examine the biological implications of detected genetic markers for PTSD.

One of the true advantages of examining heritable genetic variation is that information about DNA can be obtained from any biological sample. Furthermore, it is not necessary to be concerned with the time at which the sample is obtained relative to either the trauma exposure or the development of PTSD. This is not true for studies of epigenetics or gene expression studies that are further described below. Indeed, whereas the examination of most markers of vulnerability for PTSD might be susceptible to changes by the environment, genotype is a historical variable that precedes trauma exposure and will remain unaffected by the exposure. However without additional information about both environmental exposures and other molecular characteristics, it is not possible to ascertain whether the presence of a certain genotype has any functional significance. For this reason, genes that are identified based on a genome wide analysis should be further examined for differences in DNA methylation that may be programmed by environmental impact via epigenetic mechanisms and gene expression. In this way, it is possible to determine whether a gene variant associated with PTSD or PTSD risk is also associated with PTSD risk or pathophysiology.

\section{Genes and environment jointly predict PTSD}

A variant of genotyping studies that is particularly relevant to PTSD is the genotype-environment interaction $(\mathrm{GxE})$ design. This variant is applicable in studies where the prediction of PTSD is being made in large samples that are not chosen on the basis of trauma exposure. In the type of study described above, in which trauma survivors with PTSD are matched carefully to 
similarly exposed persons without PTSD, the GxE design may be less relevant. However, because PTSD only develops in the context of a traumatic event, it is probably never appropriate to examine only genetic main effects. GxE analyses are premised on the hypothesis that the association between a genetic variant and PTSD may depend on characteristics of the 'environment' (i.e., the trauma exposure) including such factors as type, level or severity of this exposure. In such analyses, variability in trauma characteristics is not treated as a covariate, but rather as a moderator which helps define the environmental exposure. In case-control studies where trauma survivors with PTSD are compared with those without PTSD, the influence of prior trauma exposure might very well be treated as a covariate, even though arguably, cumulative trauma exposure might also be conceptualized as an environmental factor (i.e., an environmental main effect). Indeed, exposure to trauma is not binary. Not only are levels of severity important with respect to a focal trauma, but cumulative effects of a lifetime of experiences. For example, whether the low expression allele in the promoter region of the serotonin transporter gene is associated with risk of PTSD depends on both individual-level trauma exposure severity [30] and macro-social context [33].

The possibility of observing a GxE interaction is complicated by the presence of GxE correlations, whereby genetic factors associated with PTSD may also be associated with risk of exposure to the traumatic event that is presumed to be the antecedent for PTSD [37,38]. Indeed, in classic GxE studies there is an unstated assumption that genotype and the environment - in this case trauma exposure - are independent. However, extant evidence from behavioral genetics studies clearly demonstrates that the environment shows genetic influence: the so-called nature of nurture $[39,40]$. A recent systematic review by Kendler and Baker [41] concluded: "Genetic influences on measures of the environment are pervasive in extent and modest to moderate in impact. These findings largely reflect 'actual behavior' rather than 'only perceptions'. Etiologic models for psychiatric illness need to account for the non-trivial influences of genetic factors on environmental experiences."

Indeed, if genes predict trauma exposure, then they do not necessarily interact with such events to produce a specific response. As mentioned above, twin studies have demonstrated that genetic factors influence exposure to potentially-traumatic events such as combat exposure [42] and assaultive violence [10]. These genetic effects appear to operate through personality traits.
Longitudinal investigations have found that childhood emotional adjustment and neuroticism predicted subsequent exposure to stressful life events in adulthood [43]. Similarly, research has found that childhood externalizing is prospectively associated with both risk of trauma exposure and with PTSD in adulthood [44,45]. These findings suggest gene-environment correlation for trauma may occur via an individual's personality, whereby personality characteristics influence selection of his or her environment. Innovative work that aims to overcome this limitation has focused on traumatic events, such as large-scale disasters, whose consequences are more likely to be randomly distributed across populations $[46,47]$. Because this approach may not be able to adequately capture PTSD cases that are the result of an individual's participation in the trauma exposure (e.g., perpetration of violence or participation in combat), it is necessary to use a multi-pronged approach that includes clinical or other convenience samples in addition to population-based samples. Whereas putting oneself in a dangerous situation that increases the possibility of exposure to violence may arguably have a genetic determinant, exposure to some events that are traumatic (e.g., the collapse of the World Trade Center) are more random. Thus explicitly comparing subpopulations based on trauma exposure can inform this issue.

Even more challenging for genotype-environment interaction studies of PTSD is the possibility of genetic influences on exposure to early life stress (e.g. childhood sexual abuse, physical abuse, and neglect). Childhood traumatization, particularly maltreatment, does not appear in a vacuum, but rather, in a social context that often provides fertile ground for parental abuse or neglect. Parental neglect, for example, may be a particularly strong risk factor for victimization by persons outside of the immediate family. Thus, the presence of specific gene variants might increase risk for environmental exposures through the behavior of caretakers $[41,48]$.

This is important because an association between an environment - childhood maltreatment- and an outcome - PTSD- may arise due to a third variable, namely common genetic liability. This genetic third variable confound was described in detail by DiLalla \& Gottesman [49], in responding to the current wisdom regarding the role of child abuse in the intergenerational transmission of violence [50,51]. In the specific example they critiqued, they noted that children who are abused may also have increased genetic risk for antisocial behavior, if antisocial parents are more likely 
to abuse their children and antisocial behavior is heritable. As such, the environment does not mediate the association between childhood abuse and antisocial behavior. Whether or not an association is environmentally mediated in this manner might be important for informing intervention efforts. For example, if the association between child abuse and antisocial behavior is environmentally mediated, then interventions aimed at preventing child abuse may also prevent antisocial behavior. If the association is due to a genetic third variable, then preventing abuse, although clearly important for its own sake, may well have no impact on risk for antisocial behavior.

\section{Environment modifies gene expression}

Implicit in studies of genotype in the context of PTSD is that genes may interact with the environment to produce a certain response. However, what has emerged as an even more salient concept is the idea that the environment modifies the way genes function [52]. Interestingly, trauma survivors often speak of being "transformed" by watershed life events. The study of how the environment can modify gene expression may therefore provide a biological correlate of this clinical phenomenon.

Environments do not modify genotype, but they can affect the way genes function in an enduring manner through epigenetic mechanisms (see below for description) and this can be measured in the way genes are expressed. Because gene expression can be altered by molecular mechanisms that are not epigenetic, the assessment of gene expression may not necessarily implicate epigenetics. However, a relevant epigenetic modification should alter gene expression. Thus examining epigenetic mechanisms in tandem with gene expression can help identify novel mechanisms mediated by the environment. Moreover, since genotype may preferentially influence the extent of epigenetic or gene expression changes resulting from the environment, optimally the identification of epigenetic changes or alterations in gene expression should be followed up by studies of genotype.

\section{Environmental exposures can result in epigenetic alterations}

Epigenetics refers to a transgenerationally transmissible functional change in the genome that can be due to environmental events and does not involve an alteration of sequence $[53,54]$. Although multiple types of epigenetic modifications have been identified, all involve chemical modifications that regulate chromatin structure and/or DNA accessibility, which in turn alters the transcriptional activity of the surrounding loci. Methylation - the covalent modification of DNA in which methyl groups are coupled to cytosine at $\mathrm{CpG}$ sites is perhaps the best studied of these epigenetic mechanisms, due in part to its tractability to study [55-57]. Methylation involves chemical modifications that regulate DNA accessibility, which in turn alters the transcriptional activity of the surrounding loci. In many cases, increased methylation in specific gene regions (e.g. promoter) is associated with reduced transcriptional activity and, therefore, lower gene expression. Because PTSD is influenced by environmental factors that predate exposure to a focal traumatic event, epigenetic mechanisms may be highly relevant. There is good evidence in the animal literature that DNA methylation is a mechanism operative in programming the activity of genes regulating HPA activity by early life events (i.e., differences in maternal care) [57-62] that parallel observations that early life events are associated both with the development of PTSD and the HPA axis alterations described in this condition [63-69]. Such changes result in permanent changes in hippocampal GR expression and HPA function and provide a clear molecular link between early environment and gene expression and function.

Though the similarities between effects of 'positive' maternal behaviors in rat pups (i.e., increased licking and grooming) and PTSD risk are not immediately obvious, they share in common the possibility of defining the pathways by which environmental risk factors might directly alter GR expression, thus forming a basis for individual differences in endocrine function and, perhaps, vulnerability. In theory, different cells and tissues are particularly sensitive to changes in methylation at different times during development, though in some instances, such as the development of cancer [70], DNA methylation appears to be central for the process throughout life. This is congruent with findings of a greater prevalence of PTSD following events occurring at specific developmental stages, though this disorder can develop throughout life [3,4].

Recent work suggests specific epigenetic patterns are associated with PTSD [71]. Specifically, microarraybased methylation profiles among individuals with lifetime PTSD were characterized by a preponderance of immune-related gene clusters in their unmethylated 
gene sets. Further corroboration of these findings was provided by the demonstration of significantly higher levels of antibodies to Cytomegalovirus, a typically latent herpes virus, in the PTSD affected group. Importantly, this signature of apparent immune dysregulation was also observed in pathways associated with genes showing a significant negative correlation between methylation level and number of traumatic events experienced by those with lifetime PTSD, suggesting the possibility that cumulative traumatic burden may leave a molecular footprint among those with the disorder.

\section{Environment modifies gene expression [E changes G]}

The interest in candidate gene association and epigenetic studies is premised on the hypothesis that DNA sequence variation and methylation differences are associated with downstream differences in gene expression. Gene expression is the process by which information from DNA sequence is used in the synthesis of a functional gene product. Gene expression is the fundamental process by which genotype gives rise to the phenotype. Microarray based gene-expression analysis is a method by which the expression level of a very large number of genes can be evaluated. The expression level of genes is controlled by genetic determinants, epigenetic modifications, hormonal influences, and environmental factors. The microarray technique offers the advantage of providing an unbiased survey of the mRNA expression level of nearly the entire human genome, allowing the simultaneous analysis of molecular biological pathways (e.g., genes regulating the expression of GR receptors and the downstream consequences of this regulation) that would otherwise be prohibitively resource intensive, and identifying biological processes involved in disease that were otherwise unsuspected.

A small but growing literature has provided evidence for actual changes in gene expression patterns among PTSD-affected individuals. The majority of these studies have assessed gene expression changes in RNA derived from either peripheral blood mononuclear cells (PBMCs) or whole blood. Most recently, Yehuda and colleagues [72] reported whole blood-derived gene expression levels among PTSD-affected and - unaffected individuals who had had exposure to the 9/11 attack on New York City. Differential expression was detected in 16 distinct genes, several of which are involved in signal transduction, brain and immune cell function and
HPA axis activity, including FKBP5 and signal transducer and activator of transcription 5B (STAT5B), both of which were significantly down-regulated in PTSDaffected individuals. As noted above, FKBP5 acts as an inhibitor of the glucocorticoid receptor (GR) [73] and STAT5B has been shown to delay GR nuclear translocation [74]. The detection of decreased expression in these genes is thus consistent with previous reports of higher GR activity in PTSD [75-78].

\section{Conclusion}

Genetic background may contribute to whether one is exposed to trauma and may interact with environmental exposures, but DNA function can also be modified by such exposure to these events through epigenetic pathways, resulting in alterations in gene expression. In this paper, we have argued that the combined contribution of all these factors determine susceptibility versus resilience to development of PTSD. Additionally, although biological theories of stress and fear conditioning have guided much of the research focus on the molecular biology of PTSD, it is important to broaden our conceptualizations of the response to trauma. There are still many unanswered questions regarding the extent to which genes and environmental contexts influence one another but the availability of new molecular techniques, sampling strategies, and sophisticated statistical methods ensures an almost unlimited playing field for the study of the relative contributions of genetic and environmental factors in PTSD.

\section{References}

[1] R. Yehuda, M.H. Teicher, R.L. Trestman, R.A. Levengood and L.J. Siever, Cortisol regulation in posttraumatic stress disorder and major depression: a chronobiological analysis, Biol Psychiatry 40(2) (1996), 79-88.

[2] R. Yehuda, Posttraumatic stress disorder, N Engl J Med 346 (2002), 108-114.

[3] N. Breslau, R.C. Kessler, H.D. Chilcoat, L.R. Schultz, G. Davis and P. Andreski, Trauma and posttraumatic stress disorder: The 1996 Detroit area survey of trauma, Arch Gen Psychiatry 55 (1998), 626-632.

[4] R.C. Kessler, A. Sonnega, E. Bromet, M. Hughes and C.B. Nelson, Posttraumatic stress disorder in the National Comorbidity Survey, Arch Gen Psychiatry 52 (1995), 1048-1060.

[5] A. Perkonigg, R.C. Kessler, S. Storz and H.U. Wittchen, Traumatic events and post-traumatic stress disorder in the community: prevalence, risk factors and comorbidity, Acta Psychiatr Scand 101 (2000), 46-59.

[6] R. Yehuda and J.D. Flory, Differentiating biological correlates of risk, PTSD, and resilience following trauma exposure, $J$ Trauma Stress 20(4) (2007), 435-447. 
[7] R. Yehuda and A. C.McFarlane, Conflict between current knowledge about posttraumatic stress disorder and its original conceptual basis, Am J Psychiatry 152 (1995), 1705-1713.

[8] R. Yehuda and J. LeDoux, Response variation following trauma: a translational neuroscience approach to understanding PTSD, Neuron 56 (2007), 19-32.

[9] J. Goldberg, W.R. True, S.A. Eisen and W.G. Henderson, A twin study of the effects of the Vietnam War on posttraumatic stress disorder, JAMA 263(9) (1990), 1227-1232.

[10] M.B. Stein, K.L. Jang, S. Taylor, P.A. Vernon and W.J. Livesley, Genetic and environmental influences on trauma exposure and posttraumatic stress disorder symptoms: a twin study, $A m$ J Psychiatry 159(10) (2002), 1675-1681.

[11] H. Xian, S.I. Chantarujikapong, J.F. Scherrer, S.A. Eisen, M.J. Lyons, J. Goldberg, M. Tsuang and W.R. True, Genetic and environmental influences on posttraumatic stress disorder, alcohol and drug dependence in twin pairs, Drug Alcohol Depend 61 (2000), 95-102.

[12] T.O. Afifi, G.H. Asmundson, S. Taylor and K.L. Jang, The role of genes and environment on trauma exposure and posttraumatic stress disorder symptoms: a review of twin studies, Clin Psychol Rev 30 (2010), 101-112.

[13] W.R. True, J. Rice, S.A. Eisen, A.C. Heath, J. Goldberg, M.J. Lyons and J. Nowak, A twin study of genetic and environmental contributions to liability for posttraumatic stress symptoms, Arch Gen Psychiatry 50 (1993), 257-264.

[14] K.L. Jang, M.B. Stein, S. Taylor, G.J. Asmundson and W.J. Livesley, Exposure to traumatic events and experiences: aetiological relationships with personality function, Psychiatry Res 120 (2003), 61-69.

[15] E.R. de Kloet, M. Joels and F. Holsboer, Stress and the brain: from adaptation to disease, Nat Rev Neurosci 6(6) (2005), 463-475.

[16] R. Yehuda, Status of glucocorticoid alterations in posttraumatic stress disorder, Ann N Y Acad Sci 1179 (2009), 5669.

[17] R. Yehuda, A.C. McFarlane and A.Y. Shalev, Predicting the development of posttraumatic stress disorder from the acute response to a traumatic event, Biol Psychiatry 44(12) (1998), 1305-1313.

[18] A.R, Tyrka, L.H. Price, J. Gelernter, C. Schepker, G.M. Anderson and L.L. Carpenter, Interaction of childhood maltreatment with the corticotropin-releasing hormone receptor gene: effects on hypothalamic-pituitary-adrenal axis reactivity, Biol Psychiatry 66 (2009), 681-685.

[19] A.W. Bachmann, T.L. Sedgley, R.V. Jackson, J.N. Gibson, R.M. Young and D.J. Torpy, Glucocorticoid receptor polymorphisms and post-traumatic stress disorder, Psychoneuroendocrinology 30(3) (2005), 297-306.

[20] E.B. Binder, R.G. Bradley, W. Liu, M.P. Epstein, T.C. Deveau, K.B. Mercer, Y. Tang, C.F. Gillespie, C.M. Heim, C.B. Nemeroff, A.C. Schwartz, J.F. Cubells and K.J. Ressler, Association of FKBP5 polymorphisms and childhood abuse with risk of posttraumatic stress disorder symptoms in adults, JAMA 299(11) (2008), 1291-1305.

[21] P. Xie, H.R. Kranzler, J. Poling, M.B. Stein, R.F. Anton, L.A Farrer and J. Gelernter, Interaction of FKBP5 with childhood adversity on risk for post-traumatic stress disorder, Neuropsychopharmacology 35(8) (2010), 1684-1692.

[22] J.N. Bailey, A.K. Goenjian, E.P. Noble, D.P. Walling, T. Ritchie and H.A. Goenjian, PTSD and dopaminergic genes, DRD2 and DAT, in multigenerational families exposed to the Spitak earthquake, Psychiatry Res 178(3) (2010), 507-510.

[23] D.E. Comings, D. Muhleman and R. Gysin, Dopamine D2 re- ceptor (DRD2) gene and susceptibility to posttraumatic stress disorder: a study and replication, Biol Psychiatry 40(5) (1996), 368-372.

[24] S.S. Drury, K.P. Theall, B.J. Keats and M. Scheeringa, The role of the dopamine transporter (DAT) in the development of PTSD in preschool children, J Trauma Stress 22(6) (2009), 534-539.

[25] J. Gelernter, S. Southwick, S. Goodson, A. Morgan, L. Nagy and D.S. Charney, No association between D2 dopamine receptor (DRD2) A system alleles, or DRD2 haplotypes, and posttraumatic stress disorder, Biol Psychiatry 45(5) (1999), 620-625.

[26] M. Mustapić, N. Pivac, D. Kozarić-Kovacić, M. Dezeljin, J.F. Cubells and D. Mück-Seler, Dopamine beta-hydroxylase (DBH) activity and $-1021 \mathrm{C} / \mathrm{T}$ polymorphism of $\mathrm{DBH}$ gene in combat-related post-traumatic stress disorder, Am J Med Genet B Neuropsychiatr Genet 144B(8) (2007), 1087-1089.

[27] R.H. Segman, R. Cooper-Kazaz, F. Macciardi, T. Goltser, Y. Halfon, T. Dobroborski and A.Y. Shalev, Association between the dopamine transporter gene and posttraumatic stress disorder, Mol Psychiatry 7(8) (2002), 903-907.

[28] J. Voisey, C.D. Swagell, I.P. Hughes, C.P. Morris, A. van Daal, E.P. Noble, B. Kann, K.A. Heslop, R.M. Young and B.R. Lawford, The DRD2 gene 957C $>$ T polymorphism is associated with posttraumatic stress disorder in war veterans, Depress Anxiety 26(1) (2009), 28-33.

[29] H.J. Grabe, C. Spitzer, C. Schwahn, A. Marcinek, A. Frahnow, S. Barnow, M. Lucht, H.J. Freyberger, U. John, H. Wallaschofski, H. Völzke and D. Rosskopf, Serotonin transporter gene (SLC6A4) promoter polymorphisms and the susceptibility to posttraumatic stress disorder in the general population, Am J Psychiatry 166(8) (2009), 926-933.

[30] I.T. Kolassa, V. Ertl, C. Eckart, F. Glöckner, S. Kolassa, A. Papassotiropoulos, D.J. de Quervain and T. Elbert, Association study of trauma load and SLC6A4 promoter polymorphism in posttraumatic stress disorder: evidence from survivors of the Rwandan genocide, J Clin Psychiatry 71(5) (2010), 543-547.

[31] H.J. Lee, M.S. Lee, R.H. Kang, H. Kim, S.D. Kim, B.S. Kee, Y.H. Kim, Y.K. Kim, J.B. Kim, B.K. Yeon, K.S. Oh, B.H. Oh, J.S. Yoon, C. Lee, H.Y. Jung, I.S. Chee and I.H. Paik, Influence of the serotonin transporter promoter gene polymorphism on susceptibility to posttraumatic stress disorder, Depress Anxiety 21(3) (2005), 135-139. Depress Anxiety 26(4) (2009), 309315.

[32] T.A. Mellman, T. Alim, D.D. Brown, E. Gorodetsky, B. Buzas, W.B. Lawson, D. Goldman and D.S. Charney, Serotonin polymorphisms and posttraumatic stress disorder in a trauma exposed African American population, Depress Anxiety 26(11) (2009), 993-997.

[33] K.C. Koenen, A.E. Aiello, E. Bakshis, A.B. Amstadter, K.J. Ruggiero, R. Acierno, D.G. Kilpatrick, J. Gelernter and S. Galea, Modification of the association between serotonin transporter genotype and risk of posttraumatic stress disorder in adults by county-level social environment, Am J Epidemiol 169(6) (2009), 704-711.

[34] P. Xie, H.R. Kranzler, J. Poling, M.B. Stein, R.F. Anton, K. Brady, R.D. Weiss, L. Farrer and J. Gelernter, Interactive effect of stressful life events and the serotonin transporter 5HTTLPR genotype on posttraumatic stress disorder diagnosis in 2 independent populations, Arch Gen Psychiatry 66(11) (2009), 1201-1209.

[35] S.D. Norrholm and K.J. Ressler, Genetics of anxiety and trauma-related disorders, Neuroscience 164(1) (2009), 272287. 
[36] N.R. Nugent, A.B. Amstadter and K.C. Koenen, Genetics of post-traumatic stress disorder: informing clinical conceptualizations and promoting future research, Am J Med Genet $C$ Semin Med Genet 148C(2) (2008), 127-132.

[37] S.R. Jaffee and T.S. Price, Gene-environment correlations: a review of the evidence and implications for prevention of mental illness, Mol Psychiatry 12(5) (2007), 432-442.

[38] K.C. Koenen, Nature-nurture interplay: genetically informative designs contribute to understanding the effects of trauma and interpersonal violence, J Interpers Violence 20(4) (2005), 507-512.

[39] K.S. Kendler and L.J. Eaves, Models for the joint effects of genotype and environment on liability to psychiatric illness, Am J Psychiatry 143 (1986), 279-289.

[40] R. Plomin, J.C. DeFries, G.E. McClearn and P. McGuffin, Behavioral Genetics, New York: Worth Publishers, 2008.

[41] K.S. Kendler and J.H. Baker, Genetic influences on measures of the environment: a systematic review, Psychol Med 37 (2007), 615-626.

[42] M.J. Lyons, J. Goldberg, S.A. Eisen, W. True, M.T. Tsuang, and J.M. Meyer, Do genes influence exposure to trauma? A twin study of combat, Am J Med Genet 48 (1993), 22-27.

[43] J. Van Os and P.B. Jones, Early risk factors and adult person-environment relationships in affective disorder, Psychol Medicine 29 (1999), 1055-1067.

[44] N. Breslau, V.C. Lucia and G.F. Alvarado, Intelligence and other predisposing factors in exposure to trauma and posttraumatic stress disorder: A follow-up study at age 17 years, Arch Gen Psychiatry 63 (2006), 1238-1245.

[45] K.C. Koenen, T.E. Moffitt, R. Poulton, J. Martin and A. Caspi, Early childhood factors associated with the development of post-traumatic stress disorder: Results from a longitudinal birth cohort, Psychol Med 37 (2007), 181-192.

[46] D.G. Kilpatrick, K.C. Koenen, K.J. Ruggiero, R. Acierno, S. Galea, H.S. Resnick, J. Roitzsch, J. Boyle and J. Gelernter, The serotonin transporter genotype and social support and moderation of posttraumatic stress disorder and depression in hurricane-exposed adults, Am J Psychiatry 164(11) (2007), 1693-1699.

[47] K.C. Koenen, A.B. Amstadter, K.J. Ruggiero, R. Acierno, S. Galea, D.G. Kilpatrick and J. Gelernter, RGS2 and generalized anxiety disorder in an epidemiologic sample of hurricaneexposed adults, Depress Anxiety 26(4) (2009), 309-315.

[48] S. Scarr and K. McCartney, How people make their own environments: a theory of genotype greater than environment effects, Child Dev 54 (1983), 424-435.

[49] L.F. DiLalla and I.I. Gottesman, Biological and genetic contributions to violence - Widom's untold tale, Psychol Bull 109 (1991), 125-129.

[50] C.S. Widom, Does violence beget violence? A critical examination of the literature, Psychol Bull 106 (1989), 3-28.

[51] C.S. Widom, The cycle of violence, Science 244 (1989), 160165.

[52] H.M. Abdolmaleky, C.L. Smith, S.V. Faraone, R. Shafa, W. Stone, S.J. Glatt et al., Methylomics in psychiatry: Modulation of gene-environment interactions may be through DNA methylation, Am J Med Genet B Neuropsychiatr Genet 127 (2004), 51-59.

[53] M. Nakao, Epigenetics: interaction of DNA methylation and chromatin, Gene 278 (2001), 25-31.

[54] K.L. Novik, I. Nimmrich, B. Genc, S. Maier, C. Piepenbrock, A. Olek and S. Beck, Epigenomics: genome-wide study of methylation phenomena, Curr Issues Mol Biol 4 (2002), 111128.
[55] R. Holliday, DNA methylation and epigenetic mechanisms, Cell Biophys 15(1-2) (1989), 15-20.

[56] J.E. Sutherland and M. Costa, Epigenetics and the environment, Ann N Y Acad Sci 983 (2003), 151-160.

[57] I.C. Weaver, N. Cervoni, F.A. Champagne, A.C. D'Alessio, S. Sharma, J.R. Seckl et al., Epigenetic programming by maternal behavior, Nat Neurosci 7 (2004), 847-854.

[58] D.D. Francis, F.A. Champagne, D. Liu and M.J. Meaney, Maternal care, gene expression, and development of individual differences in stress reactivity, Ann NY Acad Sci 896 (1999), $66-84$.

[59] D.D. Francis and M.J. Meaney, Maternal care and the development of stress responses, Curr Opin Neurobiol 9(1) (1999), 128-134.

[60] D. Liu, J. Diorio, B. Tannenbaum, C. Caldji, D. Francis, A. Freedman, S. Sharma, D. Pearson, P.M. Plotsky and M.J. Meaney, Maternal care, hippocampal glucocorticoid receptors, and hypothalamic-pituitary-adrenal responses to stress, Science 277(5332) (1997), 1659-1662.

[61] M.J. Meaney, Maternal care, gene expression, and the transmission of individual differences in stress reactivity across generations, Annu Rev Neurosci 24 (2001), 1161-1192.

[62] I.C. Weaver, M. Szyf and M.J. Meaney, From maternal care to gene expression: DNA methylation and the maternal programming of stress responses, Endocr Res 28(4) (2002), 699.

[63] B. Andrews, C.R. Brewin, S. Rose and M. Kirk, Predicting PTSD symptoms in victims of violent crime: the role of shame, anger, and childhood abuse, J Abnorm Psychol 109(1) (2000), 69-73.

[64] J.D. Bremner, S.M. Southwick, D.R. Johnson, R. Yehuda and D.S. Charney, Childhood physical abuse and combat-related posttraumatic stress disorder in Vietnam veterans, Am J Psychiatry 150(2) (1993), 235-239.

[65] B.S. Donovan, E. Padin-Rivera, T. Dowd and D.D. Blake, Childhood factors and war zone stress in chronic PTSD, $J$ Trauma Stress 9(2) (Apr 1996), 361-368.

[66] J.N. Epstein, B.E. Saunders and D.G. Kilpatrick, Predicting PTSD in women with a history of childhood rape, J Trauma Stress 10(4) (1997), 573-588.

[67] C. Heim and C.B. Nemeroff, The role of childhood trauma in the neurobiology of mood and anxiety disorders: preclinical and clinical studies, Biol Psychiatry 49(12) (2001), 10231039.

[68] P. Nishith, M.B. Mechanic and P.A. Resick, Prior interpersonal trauma: the contribution to current PTSD symptoms in female rape victims, J Abnorm Psychol 109 (2000), 20-25.

[69] L.Y. Zaidi and D.W. Foy, Childhood abuse experiences and combat-related PTSD, J Trauma Stress 7(1) (1994) 33-42.

[70] M. Esteller, Aberrant DNA methylation as a cancer-inducing mechanism, Annual Rev Pharmacol Toxicol 45 (2005), 629656.

[71] M. Uddin, A.E. Aiello, D.E. Wildman, K.C. Koenen, G. Pawelec, R. de Los Santos, E. Goldmann and S. Galea, Epigenetic and immune function profiles associated with posttraumatic stress disorder, Proc Natl Acad Sci U S A 107(20) (2010), 9470-9475.

[72] R. Yehuda, G. Cai, J.A. Golier, C. Sarapas, S. Galea, M. Ising, T. Rein, J. Schmeidler, B. Müller-Myhsok, F. Holsboer and J.D. Buxbaum, Gene expression patterns associated with posttraumatic stress disorder following exposure to the World Trade Center attacks, Biol Psychiatry 66(7) (2009), 708-711.

[73] G.M. Wochnik, J. Rüegg, G.A. Abel, U. Schmidt, F. Holsboer and T. Rein, FK506-binding proteins 51 and 52 differentially regulate dynein interaction and nuclear translocation of 
the glucocorticoid receptor in mammalian cells, $J$ Biol Chem 280(6) (2005), 4609-4616.

[74] E. Goleva, K.O. Kisich and D.Y. Leung, A role for STAT5 in the pathogenesis of IL-2-induced glucocorticoid resistance, $J$ Immunol 169(10) (2002), 5934-5940.

[75] M. van Zuiden, E. Geuze, M. Maas, E. Vermetten, C.J. Heijnen and A. Kavelaars, Deployment-related severe fatigue with depressive symptoms is associated with increased glucocorticoid binding to peripheral blood mononuclear cells, Brain Behav Immun 23 (2009), 1132-1139.

[76] M. van Zuiden, E. Geuze, H.L. Willemen, E. Vermetten, M.
Maas, C.J. Heijnen and A. Kavelaars, Pre-existing high glucocorticoid receptor number predicting development of posttraumatic stress symptoms after military deployment, Am J Psychiatry 168(1) (2011), 89-96.

[77] R. Yehuda, D. Boisoneau, J.W. Mason and E.L. Giller, Glucocorticoid receptor number and cortisol excretion in mood, anxiety, and psychotic disorders, Biol Psychiatry 34 (1993), 18-25.

[78] R. Yehuda, M. Lowy, S. Southwick, D. Shaffer and E. Giller, Jr., Lymphocyte glucocorticoid receptor number in posttraumatic stress disorder, Am J Psychiatry 148 (1991), 499-504. 


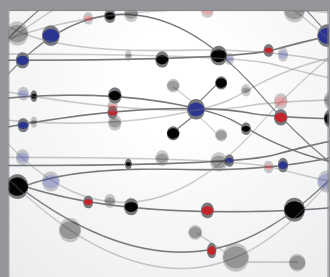

The Scientific World Journal
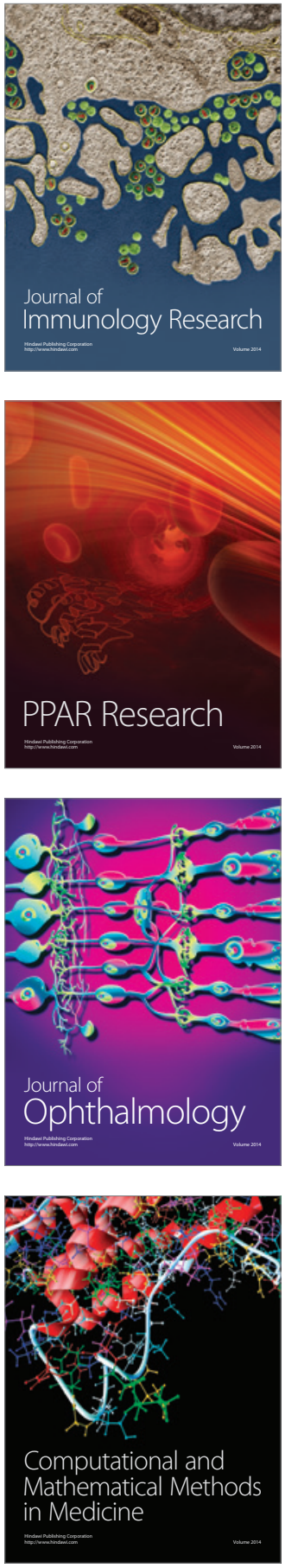

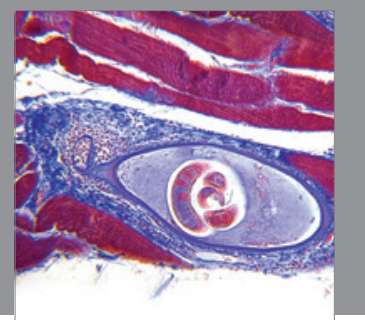

Gastroenterology

Research and Practice
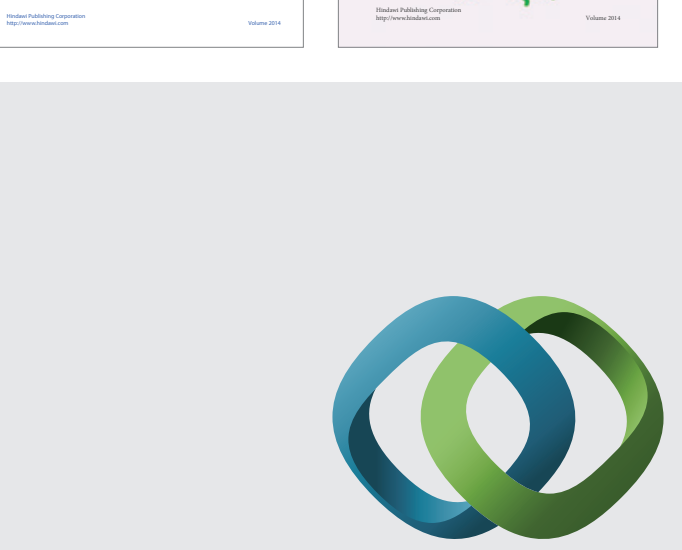

\section{Hindawi}

Submit your manuscripts at

http://www.hindawi.com
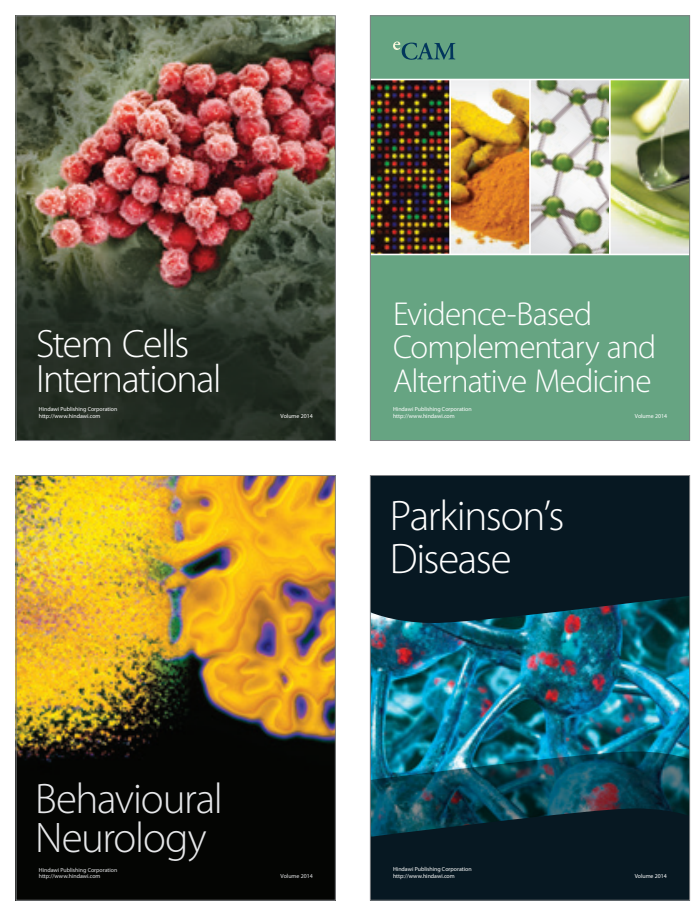

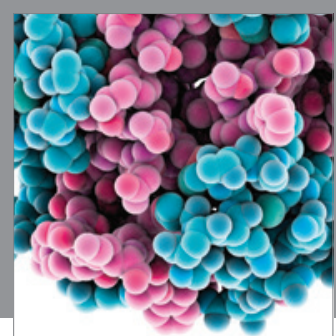

Journal of
Diabetes Research

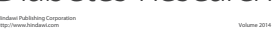

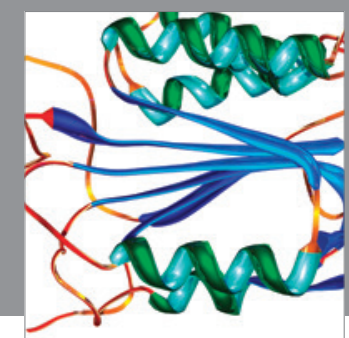

Disease Markers
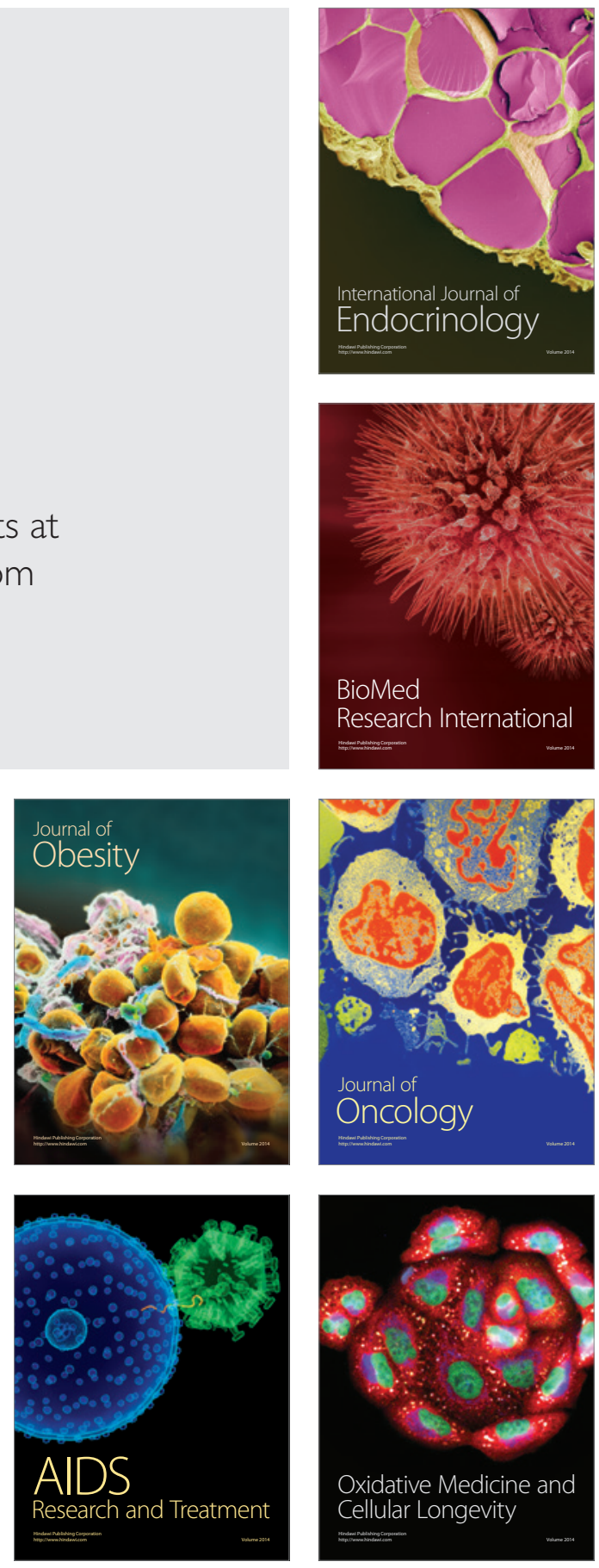